\title{
SECONDARY POSTPARTUM HAEMORRHAGE DUE TO RUPTURE OF UTERUS
}

\author{
Amna Memon, Raheel Sikandar, Fatima Memon and Farhana Saeed
}

\begin{abstract}
We report a very unusual case of secondary postpartum hemorrhage due to uterine rupture. Our case was a 23 years old lady who presented with heavy bleeding per vagina and gave history of home delivery. Rupture was most probably caused by injudicious use of oxytocic injection by a Traditional Birth Attendant at home. Surprisingly, after recovering completely from primary episode she again had heavy blood loss after 5 days. An emergency hysterectomy of the patient was performed to save the life.
\end{abstract}

KEY WORDS: Pregnancy. Uterine rupture. Hemorrhage. Oxytocin. Hysterectomy.

\section{INTRODUCTION}

Most cases of massive post-partum hemorrhage (PPH) are primary and occur within first hour after delivery. It is relatively uncommon for secondary hemorrhage to be present with blood loss of greater than $1000 \mathrm{ml}$. Incident reporting is not new in the fields of clinical medicine. Confidential inquiries into maternal deaths used to be the method to assess the quality of obstetric practice. With reduction in maternal mortality in the developed world, the new tool for assessing the obstetric care is clinical risk management (CRM). In the absence of such practice, reporting these cases in the literature keeps us well aware of these mishaps occurring in health delivery system. Uterine rupture is much more common in multiparous woman ${ }^{1,2}$. This results in hemorrhage within 24 hours of delivery, often has a dramatic presentation and it is unusual to find it being sealed with the placenta for some days and present as secondary PPH.

\section{CASE REPORT}

A 23 years old lady, para $2+1$ was admitted in maternity ward of Liaquat University Hospital (LUH), Hyderabad through casualty department at 4:30 am in November 2002. She was complaining of heavy bleeding per vagina for 1 hour. She gave history of home delivery in October ( 6 days back) followed by primary post-partum hemorrhage. A Traditional Birth Attendant (TBA) gave her intramuscular injection of oxytocin immediately before pushing her baby out. She was brought to the LUH in emergency in a state of hypotension 2 hours after delivery. A resident on duty examined her and found bleeding heavily from a cervical laceration and second-degree perineal tear. Considering it manageable in the labour room, she stitched both the tears there and shifted the patient to the ward with vaginal pack for 24 hours. She was given 2 units of blood at the time and remained well for 48 hours after removal of the vaginal pack. Then, it was decided to discharge her after getting an ultrasound for retained products of conception. Woman refused for an ultrasound scan and took discharge on request only to get admitted 3 days later with another episode of heavy bleeding. On examination, she looked anaemic. Her BP was $100 / 60 \mathrm{mmHg}$, pulse $90 \mathrm{~b} /$ minute and temperature $96^{\circ} \mathrm{F}$. Her fundal height was corresponding to $14-16$ weeks gestation and uterus was well contracted but tender. Vaginal examination revealed an infected perineal tear and a uterus of 14-16 weeks size and clear fornices. She was bleeding heavily and visualization was not possible in the labour ward. Her vaginal packing was done considering history of having a cervical tear. Baseline investigations were sent immediately, 3 units of blood were transfused. Blood complete picture showed hemoglobin $8.3 \mathrm{gm} / \mathrm{dl}$. Random blood sugar was $57 \mathrm{mg} / \mathrm{dl}$, urea $33 \mathrm{mg} \%$, BT 45 seconds and CT 8 minutes. She was shifted to operation theatre for examination under anesthesia (EUA). On EUA, she was found to have a cervical tear extending right into the body of uterus, however, limit could not be reached. On laprotomy, EUA finding was confirmed; tear was extending up to the fundus of uterus and whole placenta was found in the peritoneal cavity adherent to the site of uterine rupture. Placenta and membranes were removed and she started to bleed heavily and went into cardiac arrest because of 
hypovolumic shock. She was resuscitated and an emergency hysterectomy was performed. She was discharged on $12^{\text {th }}$ post-operative day after having a stormy post-operative period. After that, she attended out patients department twice for follow up having a phobia of vaginal pack which was put in at the time of $1^{\text {st }}$ admission and she still feels pack inside her vagina.

\section{DISCUSSION}

This is a very unusual case of uterine rupture. Rupture was most probably caused by injudicious use of oxytocic injection by TBA at home. Her $1^{\text {st }}$ admission because of post-partum hemorrhage and shock was due to uterine rupture. At that time, a resident who did not inform seniors misdiagnosed her and performed repair of cervical tear only. In spite of having a large cervical and uterine tear, she improved postoperatively and was discharged after three days of $1^{\text {st }}$ admission. Placenta and membranes present in peritoneal cavity were adherent to the rent and stopped bleeding for 48 hours. Then, she had second episode of post-partum hemorrhage, which got worst after separation of placenta form uterus. Only way of saving of her life was hysterectomy.

Complete uterine rupture can be a life-threatening emergency. Condition is rare despite the increase in caesarean section rate. Incidence reported for all pregnancies is $0.5 \%$ to $0.8 \%^{3,4}$ An important cause of rupture is previous cesarean section ${ }^{5}$. Confidential inquiries into maternal deaths in United Kingdom during 1994-1996 have reported 5 cases of uterine rupture among 7 deaths due to direct causes. Rate of uterine rupture was 2.3/ million. However, key recommendation from confidential enquiry into stillbirths and deaths in infancy ( $5^{\text {th }}$ annual report) describes that care is required in using prostaglandin for induction of labor because of the risk of hyperstimulation and uterine rupture ${ }^{6}$. Woman with previous uterine scar requires; i) antenatal management including plans for delivery and induction involving a documented discussion with an experienced obstetrician and ii) attentive intrapartum fetal and maternal surveillance in a setting where the baby can be delivered within 30 minutes. This catastrophe draws the attention towards the injudicious use of oxytocic drugs during labor and lack of involvement of senior staff in the management of post-partum hemorrhage and trauma ${ }^{7,8}$. This particular case also emphasizes on practicing CRM at obstetric units of tertiary care hospitals where deviation from standard protocol is taken as major failure. Overconfidence of a less experienced team member should always be dealt with CRM standard protocols and continuous professional development. So, we recommend that consultants should be available in the labor room all the time. Other risk factors are; previous gynaecological surgery, forceps delivery, bicornuate uterus, precipitous delivery and use of oxytocin and other uterotonics ${ }^{9-11}$. Spontaneous rupture of an unscared uterus in a woman who is not in established labor, is reported as an extremely rare complication ${ }^{12,13}$. Higher incidence of perinatal mortality and morbidity is associated with complete fetal extrusion and that significant neonatal morbidity occurs when more than 18 minutes elapse between the onsets of prolong decelerations and delivery. Farmer et al have also noted that bleeding and pain are unlikely findings (occurring) in only $3.4 \%$ and $7.6 \%$ of cases respectively ${ }^{3}$. They have reported that the most common manifestation of a scar separation is a prolonged fetal rate deceleration. Lower segment dehiscence is the commonest finding. Rupture of lower segment may extend anteriorly into the back of bladder or laterally towards the region of the uterine artery or into plexus of veins of broad ligament causing extensive hemorrhage and damage. Posterior rupture of the uterus is uncommon, but can occur with previous uterine surgery or uterine manipulation.

\section{REFERENCES}

1. Aslam M. Grand multiparty. Specialist 1994; 10:317-21.

2. Korejo R. Jafarey SN. Obstetric hysterectomy: five years experience at Jinnah Postgraduate Medical Centre, Karachi. J Pak Med Assoc 1995;45:86-8.

3. Farmer RM, Kirshbaun T, Potter D, et al. Uterine rupture during trial of labor after previous cesarean section. As J Obstet Gynecol 1991;165: 996-1001.

4. Fedorkow DM, Nimrod CA, Taylor PJ. Ruptured uterus in pregnancy: a Canadian hospital's experience. Can Med Assoc J 1987; 137: 29-9.

5. Agwu S, Llobachie GC. Ruptured uterus in England. J Obstet Gynecol 1990;10:306-11.

6. Roberts WE. Emgerent obstetric management of post-partum hemorrhage. Obstet Gynecol Clin N Am 1995; 22:283-302.

7. Lakasing L, Spencer JAD. Clinical risk management in obstetrics. In: Studd J, ed. 
Progress in Obstetrics and Gynaecology. London, Churchill Livingstone Vol.14, 2000, p.11-21.

8. Draycott $T$ and Read MD. Training obstetricians in practical skills. In: Studd J, ed. Progress in Obstetrics and Gynaecology. London, Churchill Livingstone Vol.14, 2000; p.1-10.

9. Arcangeli S, Pasquaratte MM. Gravid uterine rupture after mylosis. Obstet Gynecol 1997;85(5 part 2): 857.

10. Pushpa SS. Uterine rupture at People's Medical College Hospital, Nawabshah (1980 - 1990).
Zaidi S. (Ed). Proceedings of workshop of SOGP at Peshawar. 1993.

11. Lubna E, Nilofer R. Post-partum hemorrhage: analysis of 108 cases. J Surg Pak 2001:21-22 .

12. Sweeten KM, Graves WK, Athanassiou A. Spontaneous rupture of the unscarred uterus. Am J Obstet Gynecol 1995;172: 1851-6.

13. Langton J, Fishwick K, Kumar B, et al. Spontaneous rupture of unscarred gravid uterus at 32 weeks gestation. Hum Repord 1997;12: 2066-7.

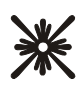

AUTHOR AFFILIATION:

Prof. Amna Memon

Department of Gynaecology and Obstetrics

Liaquat University of Medical and Health Sciences

(LUMHS), Jamshoro - Sindh.

Dr. Raheel Sikandar (Corresponding Author)

Assistant Professor

Department of Gynaecology and Obstetrics

LUMHS Jamshoro - Sindh.

Dr. Fatima Memon

Medical Student

Aga Khan University, Karachi - Sindh.

Dr. Farhana Saeed

Senior Registrar, Department of Gynaecology and Obstetrics

Liaquat University Hospital, Hyderabad - Sindh. 